\title{
Sensitivity of VIIRS polarization measurements
}

\author{
Eugene Waluschka \\ NASA/Goddard Space Flight Center/551.0, Greenbelt, Maryland 20771
}

\begin{abstract}
The design of an optical system typically involves a sensitivity analysis where the various lens parameters, such as lens spacing and curvatures, to name two parameters, are (slightly) varied to see what, if any, effect this has on the performance and to establish manufacturing tolerances. A similar analysis was performed for the VIIRS instruments polarization measurements to see how real world departures from perfectly linearly polarized light entering VIIRS effects the polarization measurement. The methodology and a few of the results of this polarization sensitivity analysis are presented and applied to the construction of a single polarizer which will cover the VIIRS VIS/NIR spectral range.
\end{abstract}

Keywords: VIIRS, polarization, ray, trace, polarizers, Bolder Vision, MOXTEK

\section{INTRODUCTION}

The Visible/Infrared Imager Radiometer Suite ${ }^{1}$ (VIIRS) instrument has had it polarization sensitivity measured three times. The first polarization test (called FP-11) was performed at Raytheon's Goleta facility in the fall of 2007, using the Polarization Source Assembly (PSA). The PSA was originally built for and used to characterize MODIS's polarization ${ }^{2,3}$. However, because this test exhibited a fair amount of erratic noise and an unexpected, and not present in any of the polarization ray trace models, detector-to-detector (D2D) linear decrease, which at the time was thought to be an artifact of the erratic noise, in polarization sensitivity in the shortest wavelength M1 band the results of this first test were not believed.

The second polarization test, at Raytheon's El Segundo facility, did not use the PSA, but instead used a large $(100 \mathrm{~cm}$ diameter) Spherical Integrating Source (SIS) and two 12 inch in diameter (large size needed to ensure a full aperture test with no vignetting) Polaroid sheets (made by Bolder Vision Optik ${ }^{4}$ ) to produce polarized light. This second test's (STR554) data showed considerably less systematic noise for all detectors on all bands over the full range of test conditions which included:

- Scan angles of $-55^{0},-45^{0},-20^{0},-8^{0},+22^{0},+45^{0},+55.5^{0}$ with respect to nadir,

- Half angle mirror (HAM) sides A and B,

- Polaroid sheets BVO777 and BVONIR needed to cover the wavelength range,

- SIS lamp levels (a few levels needed for dynamic range considerations and the prevention of saturation) and a rectangular aperture.

The plots of the data, for all of these test conditions, were by and large sinusoidal $2 \theta$ curves, as expected. This was a marked improvement over the previous PSA's data. But, again, the results showed the D2D variation and an additional feature which cast doubt on the results. The additional feature was a discrepancy between the BVO777 and BVONIR results in a wavelength range where both polarizers should have produced the same result. The BVO777 has good linear polarizance at the shorter wavelengths while the BVONIR was considered better at the longer wavelengths, but there was an overlap region where both should have worked equally well. This difference was eventually found to be due to the rectangular aperture of the SIS and the asymmetric scatter of the BVONIR polarizer. The scattered light also had a two theta, as the polarizer was rotated, intensity variation. (The motivation for using a rectangular aperture was to reduce the aperture size of the SIS and consequently increase its radiance, which was low at the shorter wavelengths.) The presences of the "false" polarization signal due to scattered light and the D2D variation, which was still perplexing, introduced sufficient doubt about the validity of the second measurement's result that a third VIIRS polarization sensitivity measurement was performed. 


\section{BETWEEN SECOND AND THIRD MEASUREMENTS}

In the time between the second and third VIIRS polarization measurements, a fair number of discussions, within the polarization working group, and modeling and lab work went into trying to understand the causes of the D2D variation and the influence of the rectangular aperture on scatter. Specifically the following was undertaken:

- the "S" and "P" reflections of the optical witness samples were re-measured with the data extending over a sufficiently large wavelength and angle of incidence range,

- this newly measured data was incorporated into Raytheon's FRED ${ }^{5}$ based polarization ray trace model,

- within the FRED model the optical surfaces had their "S" and "P" reflections spatially vary too see if this could reproduce the D2D (maybe, but not sufficiently convincing),

- a table top experiment was performed which did show conclusively that the rectangular aperture, when used with the BVONIR polarizer, does produce intensity modulated straylight which mimics a polarization signal ,

- an experiment was performed to show that a full scale test can reproduce a textbook experiment, namely measure the polarization sensitivity of a tilted glass plate in place of VIIRS. (The result did match the textbook answer to within about a $0.1 \%$ except, for an unexplained reason, at the shorter wavelength where the difference was a bit less than $0.3 \%$.)

The third polarization test, again using the SIS+Polarizer approach, did show that the second test's results were reproducible and an indication of the true polarization sensitivity of VIIRS. However, it also underscored the fact that the use of two polarizers complicates analysis and lengthens the testing of a flight instrument. Now $200 \mathrm{~mm}$ in diameter polarizers, made by MOXTEK $^{6}$, with good linear polarizance over the VIIRS VIS/NIR wavelength range are available. Using these polarizers for the VIIRS test was discussed. However, demonstrating that a subaperture test would be equivalent to a full aperture test on a flight instrument was not a viable option in view of schedule (critical path) considerations. As a result the third VIIRS polarization test was performed with the same two polarizers as in the second test (but with a circular aperture on the SIS).

Now it is certainly possible to find polarizers that work well over the entire wavelength range from 0.412 to 0.865 microns, but unfortunately not in the one foot diameter size. This resulted in the work described below.

\section{MOSAIC POLARIZER}

When testing flight instruments there is a reluctance to abandon previously used test procedures. The main reason for this is that a lot of work, at considerable expense, went into showing that, in the case of polarization testing, they do measure the polarization sensitivity of an instrument, such as VIIRS well. (Well usually means that theory and experiment agree and that the error bars in the data are sufficiently small.) Abandoning the PSA, which was used to measure the polarization sensitivity of both MODIS instruments, for the SIS+ polarizer approach was done reluctantly. It is also expected that the proposed use of a single mosaic polarizer (consisting of photo lithographically produced MOXTEK polarizers) instead of the BVO777 and BVONIR sheet polarizers will require some upfront work demonstrating both analytically and experimentally that it will be able to measure the polarization sensitivity of VIIRS no worse and more expeditiously than the two sheet polarizer approach.

The initial approach aimed at showing how well the mosaic polarizer is expected to work was done using the FORTRAN polarization raytrace model ${ }^{6}$ of VIIRS. The mosaic polarizer is currently under construction, see fig. 8 . At present the mosaic frame is built and the polarizers have been purchased and awaiting cutting into the appropriate sizes and placement into the frame. (Blank wafers have also been purchase on which to practice cutting and assembly.) Once the mosaic polarizer is built, it and the existing sheet polarizers will be used to measure the polarization sensitivity of tilted glass plates to see any differences between the various polarizers and theory. Also, it is quite possible that any testing (at Goddard) of more complicated optical systems will show that all three polarizers (BVO777, BVONIR and MOXTEK mosaic) produce, in the appropriate wavelength ranges, the same results and hopefully this will show that the mosaic polarizer is indeed good enough for any future VIIRS polarization testing. However, it is certainly possible, time and money permitting, that all three polarizers will be used to test VIIRS. 
The following sections will state the VIIRS polarization requirement, describe the polarization test, show a bit of useful mathematical formalism, briefly describe the PRT model, present a few sensitivity results and describe the current status of the mosaic polarizer.

\section{THE POLARIZATION REQUIREMENT}

For reference the VIIRS's instrument polarization requirement read as follows:

"The VIIRS Sensor linear polarization sensitivity of the VIS and NIR bands shall be less than or equal to the values indicated in Tablel for scan angles less than 45 degrees off Nadir.

The VIIRS sensor linear polarization sensitivity shall be measured within a characterization uncertainty of $0.5 \%$ (one sigma) for scan angles less than 55.84 degrees off nadir.

The angle (phase) of the polarization sensitivity will be calculated from the same data that is used to determine the magnitude of the polarization sensitivity. The uncertainty of the phase angle is proportional to the uncertainty of the polarization amplitude. The contractual definition of the instruments polarization sensitivity is giving by the well known equ.1."

$$
\frac{I_{\max }-I_{\min }}{I_{\max }+I_{\min }} \leq 0.03
$$

Table 1. Polarization sensitivity requirements

\begin{tabular}{|c|c|c|}
\hline Band & $\begin{array}{c}\text { Center } \\
\text { Wavelength } \\
(\boldsymbol{\mu} \mathbf{m})\end{array}$ & $\begin{array}{c}\text { Maximum } \\
\text { Polarization } \\
\text { Sensitivity }\end{array}$ \\
\hline M1 & 0.412 & $3 \%$ \\
\hline M2 & 0.445 & $2.5 \%$ \\
\hline M3 & 0.488 & $2.5 \%$ \\
\hline M4 & 0.555 & $2.5 \%$ \\
\hline I1 & 0.640 & $2.5 \%$ \\
\hline M5 & 0.672 & $2.5 \%$ \\
\hline M6 & 0.746 & $2.5 \%$ \\
\hline I2 & 0.865 & $3 \%$ \\
\hline M7 & 0.865 & $3 \%$ \\
\hline
\end{tabular}

Prior to describing the sensitivity analysis and results a description of the VIIRS polarization measurement test configuration, which in a clean room and is very similar to a radiometric calibration, with VIIRS looking direcely into a SIS, but in a polarization measurement there is a rotating polarizer between the SIS and VIIRS.

\section{TEST CONFIGURATION}

Fig. 1 shows schematically the VIIRS's polarization test configuration ${ }^{7}$

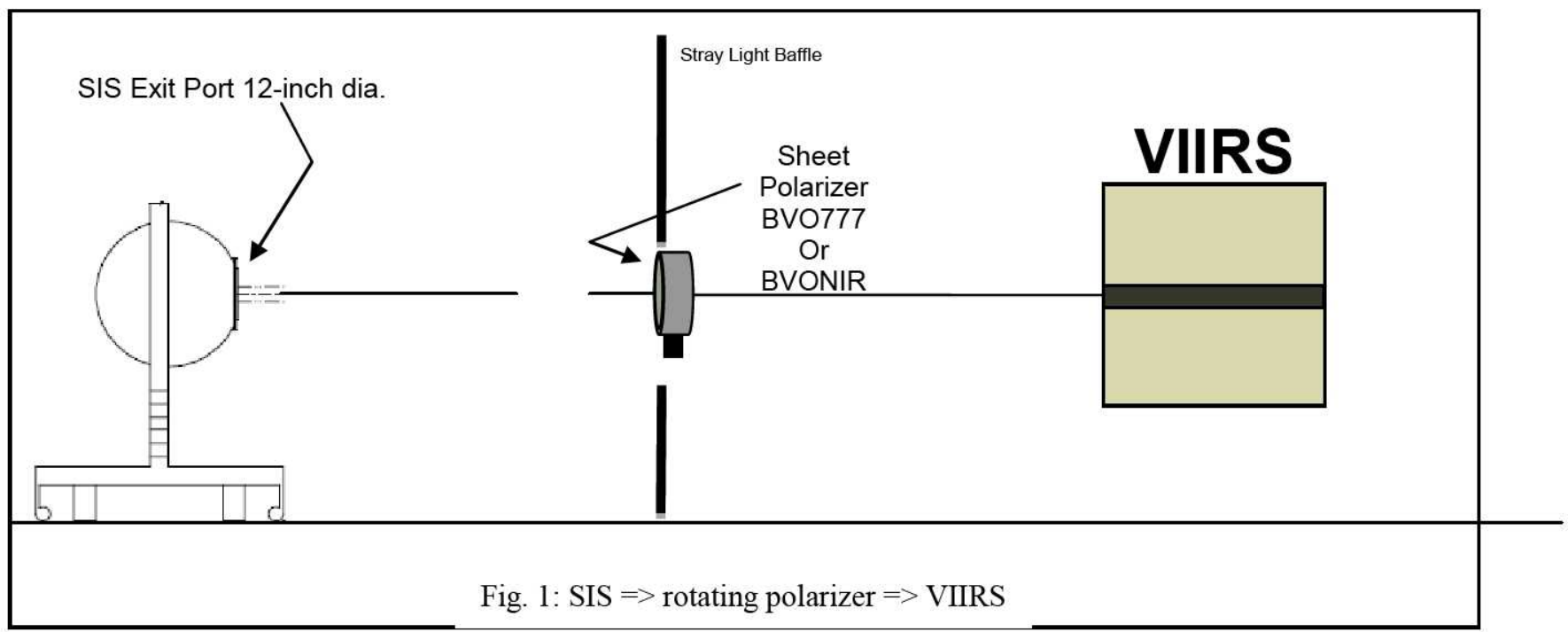


The source of unpolarizer light is a large aperture (rectangular in the second and circular in the third polarization measurement) spherical integration source. A useful shorthand description of the test is the following:

$$
\mathrm{SIS}=>\text { rotating polarizer }(\theta)=>\text { VIIRS }
$$

The polarizer was about 12 inches in diameter. The sizes of the SIS and the polarizer apertures are large enough to ensure that there would be no vignetting of the field of view of the telescope, with its approximately 8 inch aperture, as it viewed the polarizer while rotating through about one degree during a polarization measurement. (The test as you fly approach has the telescope rotating during the polarization testing.)

\section{MATHEMATICAL OVERVIEW}

The object of the experiment is to record, for each detector on the VIIRS VIS/NIR focal plane, the variation in incident light as the polarizer is rotated through $0^{\circ}-360^{\circ}$ in $15^{\circ}$ steps. In terms of Stokes and Mueller matrices we have, equ. 2 for each detector

$$
S_{\text {final }}(\theta)=A \times R^{-1}(\theta) \times P \times R(\theta) \times S_{\text {initial }}
$$

where $S_{\text {initial }}$ is the Stokes vector describing the light leaving the SIS, $R(\theta)$ is a Mueller rotation matrix, $P$ is the Mueller matrix of the sheet polarizer, $A$ is the Mueller matrix of the VIS/NIR optics specific and (slightly) different for each detector and $S_{\text {final }}(\theta)$ the Stokes vector reaching a particular detector. Showing the matrix components we have equ. 3.

$$
\left(\begin{array}{l}
I(\theta) \\
Q(\theta) \\
U(\theta) \\
V(\theta)
\end{array}\right)=\left(\begin{array}{llll}
a_{11} & a_{12} & a_{13} & a_{14} \\
a_{21} & a_{22} & a_{23} & a_{24} \\
a_{31} & a_{32} & a_{33} & a_{34} \\
a_{41} & a_{42} & a_{43} & a_{44}
\end{array}\right)\left(\begin{array}{cccc}
1 & 0 & 0 & 0 \\
0 & \cos (2 \theta) & -\sin (2 \theta) & 0 \\
0 & \sin (2 \theta) & \cos (2 \theta) & 0 \\
0 & 0 & 0 & 1
\end{array}\right)\left(\begin{array}{cccc}
p_{x}^{2}+p_{y}^{2} & p_{x}^{2}-p_{y}^{2} & 0 & 0 \\
p_{x}^{2}-p_{y}^{2} & p_{x}^{2}+p_{y}^{2} & 0 & 0 \\
0 & 0 & 2 p_{x} p_{y} & 0 \\
0 & 0 & 0 & 2 p_{x} p_{y}
\end{array}\right)\left(\begin{array}{cccc}
1 & 0 & 0 & 0 \\
0 & \cos (2 \theta) & \sin (2 \theta) & 0 \\
0 & -\sin (2 \theta) & \cos (2 \theta) & 0 \\
0 & 0 & 0 & 1
\end{array}\right)\left(\begin{array}{l}
S_{1} \\
S_{2} \\
S_{3} \\
S_{4}
\end{array}\right)
$$

where $p_{x}$ and $p_{y}$ are the maximum and minimum transmission, at a particular wavelength, of the polarizer to incident linearly polarized light. If we assume that the light leaving the SIS is sufficiently unpolarized then we can, to a fair approximation assume $S_{2}=S_{3}=S_{4}=0$ and equ. 3 simplifies to equ. 4

$$
\left(\begin{array}{l}
I(\theta) \\
Q(\theta) \\
U(\theta) \\
V(\theta)
\end{array}\right)=\left(\begin{array}{l}
S_{1}\left[\left(a_{12} \cos (2 \theta)+a_{13} \sin (2 \theta)\right)\left(p_{x}^{2}-p_{y}^{2}\right)+a_{11}\left(p_{x}^{2}+p_{y}^{2}\right)\right] \\
S_{1}\left[\left(a_{22} \cos (2 \theta)+a_{23} \sin (2 \theta)\right)\left(p_{x}^{2}-p_{y}^{2}\right)+a_{21}\left(p_{x}^{2}+p_{y}^{2}\right)\right] \\
S_{1}\left[\left(a_{32} \cos (2 \theta)+a_{33} \sin (2 \theta)\right)\left(p_{x}^{2}-p_{y}^{2}\right)+a_{31}\left(p_{x}^{2}+p_{y}^{2}\right)\right] \\
S_{1}\left[\left(a_{42} \cos (2 \theta)+a_{43} \sin (2 \theta)\right)\left(p_{x}^{2}-p_{y}^{2}\right)+a_{41}\left(p_{x}^{2}+p_{y}^{2}\right)\right]
\end{array}\right)
$$

The measured intensity is given by equ. 5 .

$$
I(\theta)=S_{1}\left[\left(a_{12} \cos (2 \theta)+a_{13} \sin (2 \theta)\right)\left(p_{x}^{2}-p_{y}^{2}\right)+a_{11}\left(p_{x}^{2}+p_{y}^{2}\right)\right]
$$

With the polarizer rotated in 15 degree steps,

$$
\theta=0^{\circ}, 15^{\circ}, 30^{\circ}, \ldots, 360^{\circ}
$$

this results in 25 data points which are subsequently converted to as many equations 


$$
\begin{aligned}
& I\left(\theta_{1}\right)=S_{1}\left[\left(a_{12} \cos \left(2 \theta_{1}\right)+a_{13} \sin \left(2 \theta_{1}\right)\right)\left(p_{x}^{2}-p_{y}^{2}\right)+a_{11}\left(p_{x}^{2}+p_{y}^{2}\right)\right] \\
& I\left(\theta_{2}\right)=S_{1}\left[\left(a_{12} \cos \left(2 \theta_{2}\right)+a_{13} \sin \left(2 \theta_{2}\right)\right)\left(p_{x}^{2}-p_{y}^{2}\right)+a_{11}\left(p_{x}^{2}+p_{y}^{2}\right)\right] \\
& \text { - } \\
& I\left(\theta_{25}\right)=S_{1}\left[\left(a_{12} \cos \left(2 \theta_{25}\right)+a_{13} \sin \left(2 \theta_{25}\right)\right)\left(p_{x}^{2}-p_{y}^{2}\right)+a_{11}\left(p_{x}^{2}+p_{y}^{2}\right)\right]
\end{aligned}
$$

which form an over determined set of simultaneous equations and which can be solved for the three unknowns $a_{11}, a_{12}$ and $a_{13}$. The degree of linear polarization can be determined from measurements using equ. 1 or equ. 8

The phase is determined from

$$
D O L P=\frac{\sqrt{a_{12}^{2}+a_{13}^{2}}}{a_{11}}
$$

$$
\text { phase }=0.5 \cdot \tan ^{-1}\left(\frac{a_{12}}{a_{13}}\right)
$$

In practice, a Fourier transform approach is used analyze the data. Equ. 10 is fit to the data

$$
I(\theta)=I_{0}+\sum_{n=1}^{4}\left(A_{n} \cos (n \theta)+B_{n} \sin (n \theta)\right)
$$

and $I_{0}, A_{2}, B_{2}$ are then used in equ. 8 and 9 . With this approach the DOLP is subsequently increased to take into account the inefficiency of the polarizer to produce perfectly linearly polarized light. The phase with this approach has typically not been adjusted.

It should be noted that in the above discussion the polarizer is assumed to be homogeneous, that is $p_{x}$ and $p_{y}$ are constant over the entire polarizer clear aperture. In actuality the BVO777, the BVONIR and the mosaic polarizer will have spatial variations. The effects of these non-uniformities on the polarization sensitivity were examined using the FORTRAN PRT model, presented in the following section.

\section{FORTRAN POLARIZATION RAY TRACE MODEL}

Fig. 2, shows, schematically, the polarizer and all optics in the VIS/NIR model. Typically a1 5 by 15 bundle of parallel rays, starting before the polarizer and filling the primary mirror's clear aperture is traced through the optics coming to a focus at a detector. The rotating telescope consists of three powered elements and a flat fold mirror which directs the light onto the rotating "half-angle" mirror (HAM), which is a two sided rotation fold mirror. The half angle mirror, rotating at half the speed of the telescope de-rotates the light beam and directs it into the aft optics. It should be noted that the HAM is the only optic with a varying -during scanning - angle of incidence and this cause a scan angle dependence in the polarization sensitivity of VIIRS. The aft-optics consists of fold mirror \#2 immediately following the HAM, then four reflecting elements, then dichroic \#1 which reflects the visible and near-IR light toward the focal plane filter assembly, which is immediately above the detectors, fig. 3 .

This FORTRAN PRT model ${ }^{8}$ does not have the most up-to-date measured optical surface reflectances. Raytheon's FRED model does have the most up-to-date measured surface reflectace data. However, both models predict approximately the same polarization sensitivity for VIIRS and neither model shows the measured D2D variation to any extent. As both models give approximately the same results, it is reasonable to assume that either model can be used to predict small changes in VIIRS's polarization sensitivity due to small changes in the polarizer transmission characteristics. With this in mind the FORTRAN model with the original coatings is the starting point for the sensitivity analysis, the baseline case. 


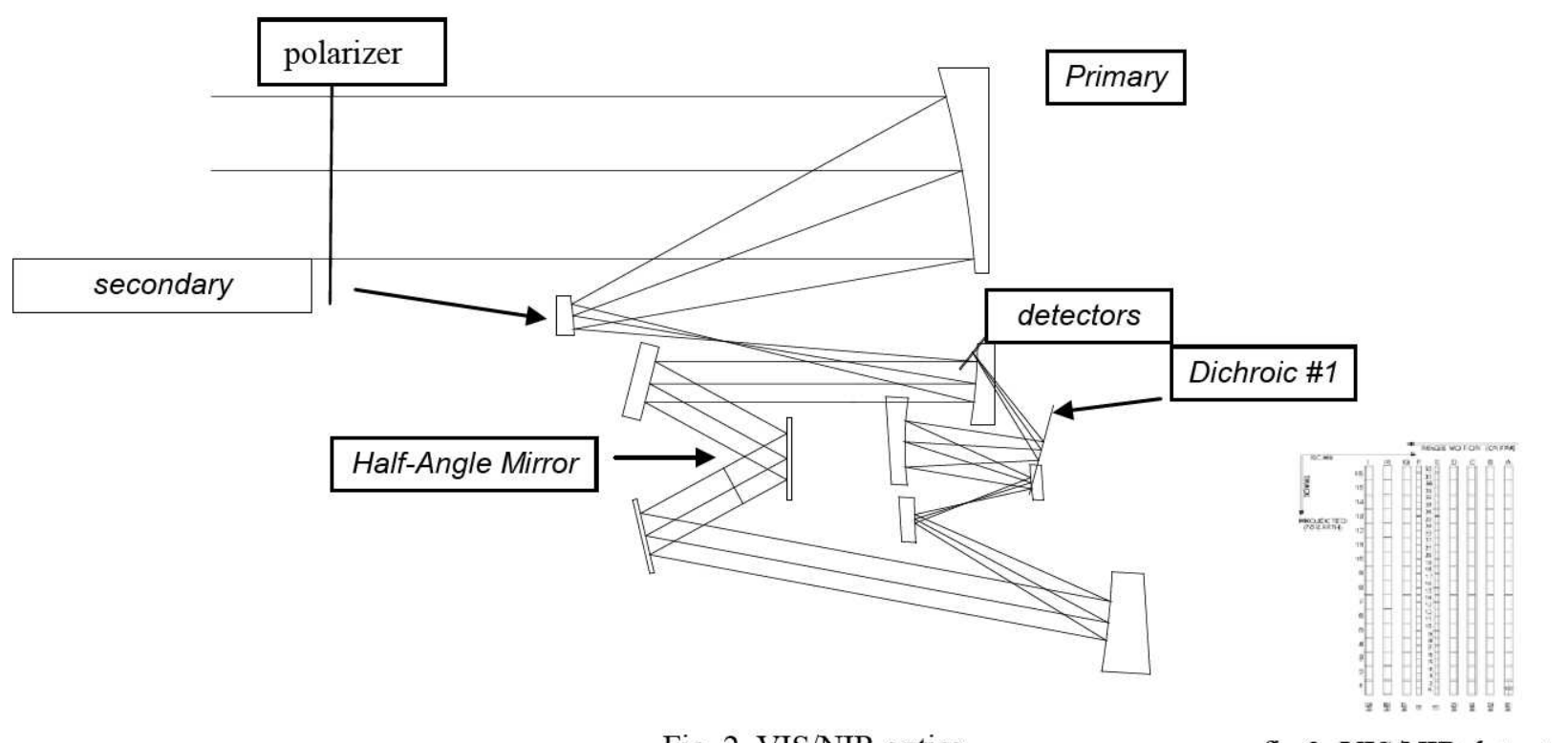

Fig. 2. VIS/NIR optics.

fig.3: VIS/NIR detectors

\section{BASELINE}

The baseline PRT model assumes that unpolarized light, sampled on a regular grid fig. 4, leaves the SIS and becomes fully linearly polarized after passing through the polarizer. The baseline case models a perfectly homogeneous polarizer with no spatial variations of any kind. The model has all nine bands on the VIS/NIR focal plane, but only three, fig.3, top, middle and bottom detectors in each band. Fig. 5 shows the outline of the portion of the polarize seen by each three detectors in each of the nine bands.

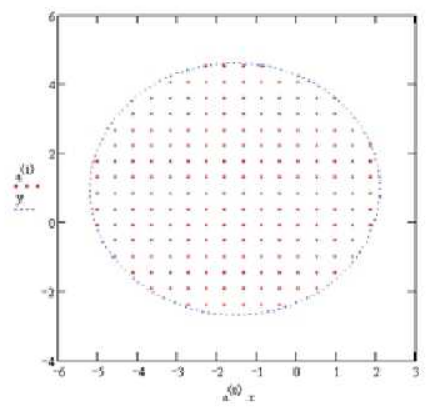

fig.4: Ray grid

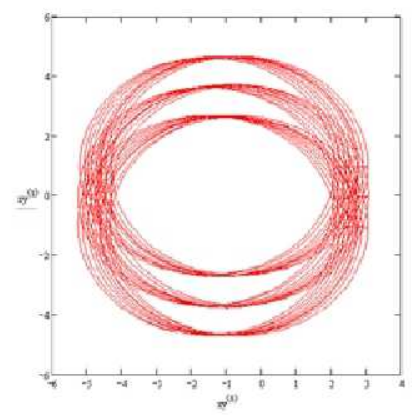

fig.5: Footprints on polarizer

The computation proceeds by rotating the polarizer through 10 degree steps and stepping through all of the in-band wavelengths in $0.001 \mathrm{~nm}$ steps. At the end of each computation we have for each band and the three detectors in each band a table of numbers showing how the transmission of the optics changes as the entering linearly polarizer light rotates. Fig.6 shows only one detector's baseline polarization response for one scan angle. 


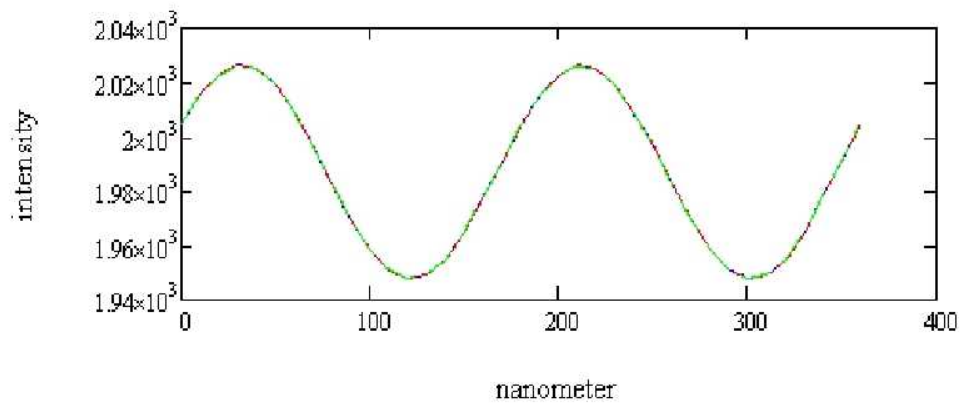

Fig 6: One detectors baseline

The computation is repeated for the $-45^{\circ},-22.5^{\circ}, 0^{\circ},+22.5^{\circ},+45^{\circ}$ telescope scan angles resulting in 135 files. (Three detectors per band times nine bands times five scan angles equals 135.) All of the data in these files constitutes this model's baseline polarization sensitivity. If plotted, the sinusoidal variations would resemble fig.6. Their function form is given by equ. 10, which is similar to equ. 5 , but with $\left(p_{x}^{2}-p_{y}^{2}\right)=\left(p_{x}^{2}+p_{y}^{2}\right)=1$ for a perfect polarizer.

$$
I(\theta)=S_{1}\left[a_{11}+a_{12} \cos (2 \theta)+a_{13} \sin (2 \theta)\right]
$$

Using the procedure in section 6 to determine the 135 sets of three Mueller matrix elements $a_{11}, a_{12}, a_{13}$ and plotting what have become known as the "two theta Fourier coefficients" $a_{12}$ vs. $a_{13}$ we get 135 points as shown in fig. 7 . In this kind of plot the distance of any point from the origin is proportional, see equ. 8 , to the degree of polarization. The phase angle, equ. 9, is less evident, but can also be visually inferred. The five (corresponding to the five telescope view directions) sets of three (corresponding to the top, middle and bottom detectors) points towards the upper right in fig.7 are furthest way from the origin indicating that band M1, with the shortest wavelength has the most polarization sensitivity, as is also observed in test.

These 135 points constitute the VIIRS modeled baseline response to uniform illumination of the full aperture with linearly polarized light and nominal coatings

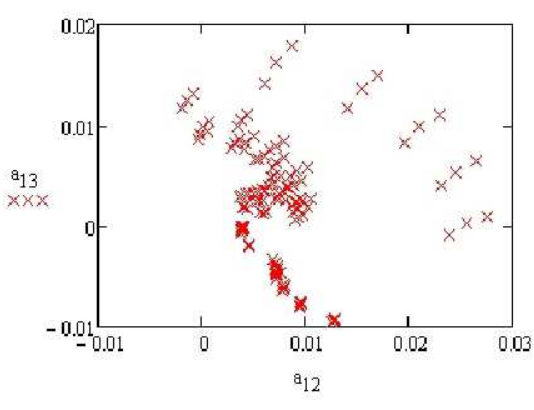

Fig. 7: 135 baseline points on the optics. The section below shows how these points move when the polarizer is no longer full aperture or is not uniform, but has spatial variations.

\section{SUBAPERTURE, LINEAR GRADIENT AND MOSAIC}

An extensive polarization sensitivity analysis, using the computer code FRED, was performed by Raytheon. The goal in that effort was to find the causes of the D2D. The approach was to incorporate newly measured witness piece measured data and also to vary the "S" and "P" spatial reflectances of the optical surfaces inside of VIIRS. Goddard's polarization sensitivity analysis, which was started toward the end of Raytheon's modeling work, while hoping to find the elusive causes for the D2D variation, had more in mind the construction of mosaic polarizer using MOXTEK polarizers. The initial plan was to cut the circular wafers into five inch squares and edge bond them. This approach would result in a thin bond line, but would produce a thin, fragile and rather large optic which requires great care in handling. With the thought that in all likelihood this edge bonded mosaic would break at the least opportune time, as during VIIRS flight model 2 polarization testing, a more robust approach is being taken. A

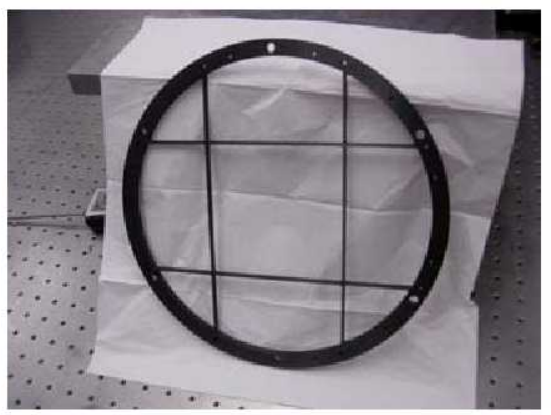

Fig. 8: Mosaic rib structure 
galvanized aluminum frame, fig. 8 , is constructed to hold the thin MOXTEK polarizers. As can be seen the ribs of this frame are noticeable.

Prior to the third VIIRS polarization test there was some discussion of a subaperture test. The reason for this is that one of the problems of measuring VIIRS's polarization using the SIS+ polarizer approach was the lack of large sheet polarizers which polarized light well over the VIS/NIR wavelength range. A smaller diameter, such as the MOXTEK 200mm clear aperture polarizer does work well over that spectral range, but it would have been a subaperture test and most likely would have required that the telescope not rotate, but stare directly at the smaller polarizer. Even though its use was precluded for a number of reasons, it is interesting to note that the PRT model predicts that a polarizer which is $1 / 2$ of the VIIRS clear entrance aperture diameter would produce a set of coefficients $a_{11}, a_{12}$ and $a_{13}$ not much different from a full aperture test. Fig. 9 shows this result as a plot of 135 differences in the two theta coefficients $\left(a_{12}^{\text {baseline }}-a_{12}^{\text {half aperture }}, a_{13}^{\text {baseline }}-a_{13}^{\text {half aperture }}\right)$. Stating the percent change, instead of just the difference, in the coefficients can be misleading because points near the origin are likely to have a greater relative change than points further away.

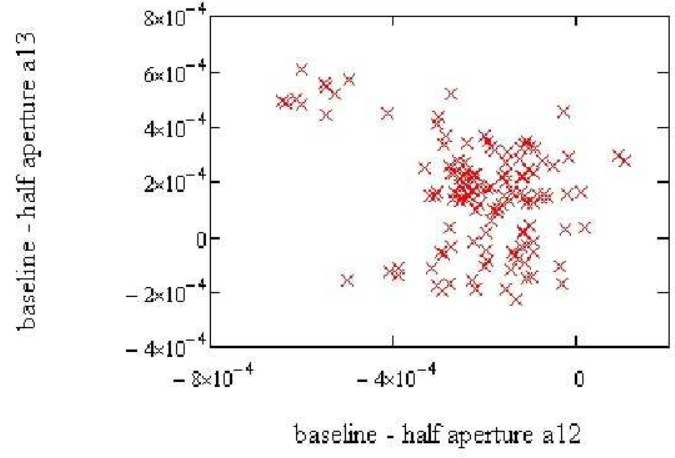

Fig. 9: Half aperture difference from Baseline

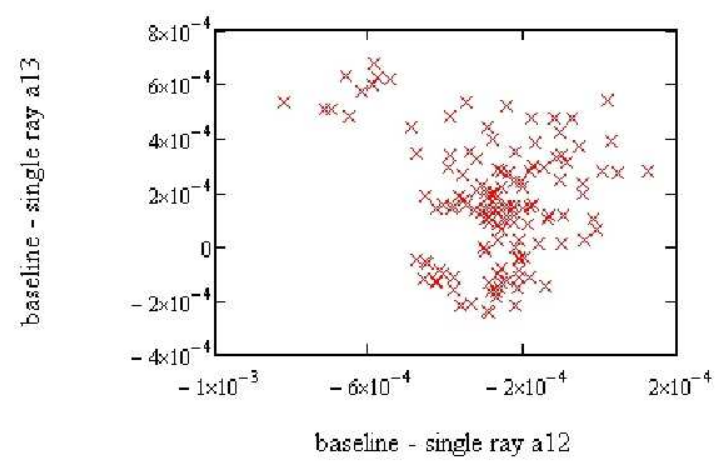

Fig 10: Single ray difference from Baseline

It is also noteworthy that a single chief ray's, the equivalent of thin pencil of light, PRT result, shown in fig. 10, does not differ much from the baseline. However, because of the requirement that the uncertainty in the measurement not be greater than $3 \%$ and sub aperture testing does increase the uncertainty, it is unlikely that partial aperture testing will be used to characterize VIIRS flight unit 2, but it should be kept in mind as a diagnostic tool should the need arise to determine either a detector-to-detector variation or some other perplexing observation.

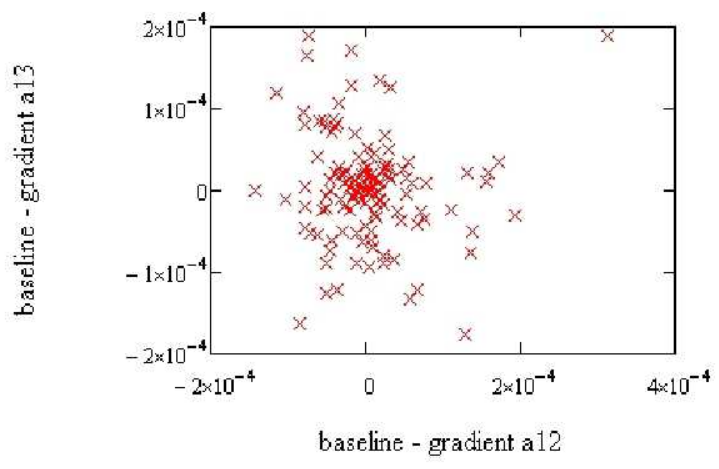

Fig. 11: linear gradient difference from Baseline

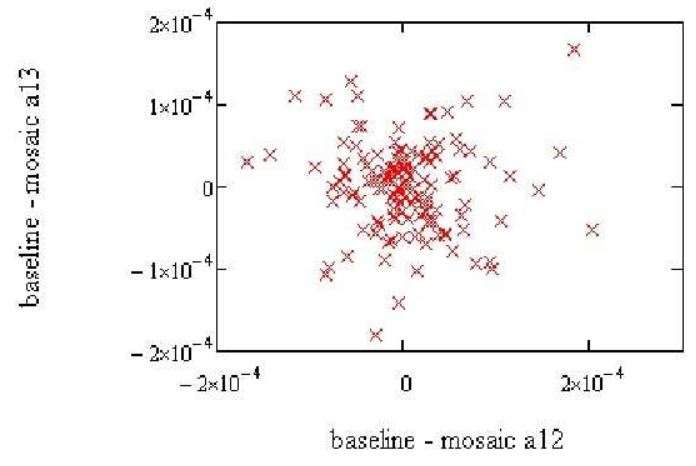

Fig 12: Mosaic - Baseline

Another topic discussed in connection with the 12 inch diameter sheet polarizer was their spatial uniformity. The sheet polarizers are not perfectly spatially uniform. If we assume that there is a $1 / 4 \%$ per inch transmission gradient, a not unreasonable assumption, then with this polarizer variation the computed difference in polarization sensitivity from the baseline's perfectly uniform polarizer, is not great as shown in fig. 11 . Not shown here, but the $1 \%$ per inch transmission gradient case is, in magnitude similar to the $1 / 4 \%$ case. 
Lastly, fig. 12 shows the modeled change from the baseline case when a mosaic polarizer, with obscuring ribs and with a $1 \%$ per 5 inch transmission gradient in each pane. The difference in the set of computed coefficients $a_{11}, a_{12}$ and $a_{13}$ for this case is again small. Hopefully this is an indication that any future polarization measurement of VIIRS using the mosaic polarizer and the BVO777 and BVONIR polarizers will produce identical results in all three cases over the wavelength range where all three polarizer are presumed good.

\section{CONCLUSION}

A fair amount of work went into understanding the results and the uncertainties of the VIIRS polarization measurements. One of the tasks performed was a series of polarization ray traces specifically looking at the effects of spatial non-uniformities in the polarizers on the measurement. The results of this work indicate that moderate spatial transmission variations in the polarizers have only a small effect on the measurement and that a single mosaic polarizer with a rib structure, but with good polarization characteristics over the needed wavelength range should determining VIIRS's polarization sensitivity to within the required measurement uncertainty limits. Construction of the mosaic polarizer is ongoing. Work and testing of the mosaic should be complete in time for VIIRS flight model 2 polarization testing. Whether-or-not it will be used remains to be seen. Preliminary tests hopefully will show that its use in VIIRS polarization testing is a viable option.

\section{ACKNOWLEDGMENTS}

Thanks go to the VIIRS polarization working group whose members (can be found scattered across the United States) have been working on improving and understanding VIIRS's polarization measurement for a number of years. The result of all of this protracted activity is a good deal of insight into the activities surrounding the testing of a flight instrument and an improvement in the testing. It's a pleasure working with you. Thanks go to Eric Fest for corroborating some of the partial aperture computations using FRED. Thanks also go to Brendan McAndrew for setting in motion the construction of the mosaic polarizer. Without his help the mosaic polarizer would probably still be a sketch. Special thanks go to Bruce Guenther and the Integrated Products Office's Karen St.Germain for their continued support. This work was funded by NOAA and NASA.

\section{REFERENCES}

1. http://www.ipo.noaa.gov/Technology/viirs summary.html

2. M.Pavlov, "Y15191 Summary of the PSA characterization", May 11,2004

3. J. Young, B. Sornsin, S. Engelman and I, Lorkovic, "Y22558 Polarized Source Assembly (PSA) Acceptance Testing Review for VIIRS", March 30, 2007

4. http://www.boldervision.com/

5. Raytheon report VIIRS FU1 Polarization Sensitivity Analysis

6. http://www.moxtek.com/

7. Raytheon report Performance Verification Report - VIIRS FU1 Polarization Sensitivity

8. E. Waluschka, K. Voss, D. Moyer, G. Meister, and L. Liao, "VIIRS ZEMAX and FORTRAN polarization models", Proc. SPIE 6677, (2007) 